\title{
IMPROVEMENT OF EXISTING AND DEVELOPMENT OF FUTURE COPERNICUS LAND MONITORING PRODUCTS-THE ECOLASS PROJECT
}

\author{
E.Sevillano Marco, D.Herrmann, K. Schwab , K. Schweitzer, R. Almengor, F. Berndt, C. Sommer, M. Probeck \\ - (eva.sevillanomarco, david.herrmann, katharina.schwab, kathrin.schweitzer, roger.almengor, fabian.berndt, \\ carolin.sommer, markus.probeck)@gaf.de
}

GAF AG, Munich, Germany

KEY WORDS: Copernicus Land Monitoring Service, ECoLaSS, Earth Observation, DIAS, HRL, LCLU, Sentinel, time series analysis.

\begin{abstract}
:
The Horizon 2020 project ECoLaSS (Evolution of Copernicus Land Services based on Sentinel data) contributes to improving existing and developing next-generation Copernicus Land Monitoring Service (CLMS) products. The High Resolution Layers (HRLs) are currently produced in regular 3-year intervals at 10-20 meter spatial resolution for 39 European countries (EEA 39). Evolving scientific developments and user requirements are continuously analysed in a close stakeholder interaction process with the European Entrusted Entities (EEE), targeting a future pan-European roll-out of new/improved CLMS products and assessing transferability to global applications. Products and methods are being prototypically demonstrated. Representative sites $\left(60,000-90,000 \mathrm{~km}^{2}\right)$ were selected, covering boreal, Mediterranean, steppic, Atlantic, alpine and continental conditions. Improvements comprise yearly updates of enhanced dominant leaf types and tree cover change layers, better-quality permanent grassland classification and use categorisation. Novel products target agriculture products (i.e., crop mask, crop types). Temporal analysis, based on optical (Sentinel-2) and SAR (Sentinel-1) satellite data, makes use of temporal feature descriptors (multiple temporal statistical metrics) derived from spectral bands and indices (e.g., VV/VH ratio and NDVVVH from SAR data and NDWI, NDVI, Brightness and IRECI from optical data). Overall accuracies range from $77-98 \%$. Rigorous benchmarking is applied to assess the prototypes' operational readiness and technical maturity for integration into the CLMS architecture.
\end{abstract}

\section{INTRODUCTION}

The Copernicus Programme, managed and coordinated by the European Commission (EC) and implemented in partnership with the European Space Agency (ESA), member states and various EU agencies, offers services mainly based on Earth Observation (EO) data provided by ESA through the Copernicus Space Component. Complementing the operational-phase implementation, the Horizon 2020 project ECoLaSS (Evolution of Copernicus Land Services based on Sentinel data) is being conducted from 2017-2019 and aims at developing and prototypically demonstrating selected innovative products and methods as candidates for future next-generation operational Copernicus Land Monitoring Service (CLMS) products of the pan-European and Global Components, assessing the operational readiness of such candidate products and suggesting an implementation schedule. This shall enable the key CLMS stakeholders (i.e. mainly the Entrusted European Entities (EEEs) EEA and JRC) to take informed decisions on potential procurement as (part of) the next generation of Copernicus Land services from 2020 onwards.

Among the key products of the pan-European component of the CLMS are the so-called High Resolution Layers (HRLs), which are thematic products currently targeting land cover characteristics of five main classes: Imperviousness, Forest, Grassland, Water/Wetness and Small Woody Features (HRL reference year 2015). Most of these layers are produced in regular 3-year intervals from multi-temporal EO data at 10-20 meter spatial resolution for 39 European countries (EEA 39). Further similar and other products (such as a HR Arable Land Layer) are in the debate. The EEEs published some of the next generation HRLs products and services specifications, which include improved product provisions, production timeliness, etc.

Rapidly evolving scientific developments as well as user requirements are continuously analysed in a close stakeholder interaction process, addressing the panEuropean roll-out of new/improved CLMS products, and exploring a potential transferability to global applications. The prototyping implementations in ECoLaSS acknowledge and meet such interests.

At this stage in project development, the prototypes presented here account for identified needs and are in line with the user requirements compiled: higher interest in local components (i.e., implying higher resolution products), higher update frequencies and incremental updates, change detection, interest in Copernicus satellite data (mostly Sentinel-2), and in particular in production based on optics and SAR at $10 \mathrm{~m}$ derived from Sentinel. Specific products demanded are addressing the HRLs (e.g., improved Grassland identification, new Grassland Use Intensity product, Forest cover and change layers), enhanced CORINE Land Cover (i.e., CLC+ specifications to be published in 2019) and new services appealing to Agriculture (e.g., crop mask, crop types, yield products) and cross-cutting generic products (e.g., vegetation indicators, phenology, biophysical variables). The proof-ofconcept demos match the HRLs 2018 iteration.

Methodological implementation is based on improved EO data pre-processing, multisensor integration, state of the art time series analysis and sophisticated change detection 
concepts. ECoLaSS makes full use of dense time series of High-Resolution (HR) Sentinel-2 optical and Sentinel-1 Synthetic Aperture Radar (SAR) data, complemented by ancillary optical data (e.g., PROBA-V, Landsat 8, and additional VHR data from the ESA Data Warehouse DWH) and elevation data (e.g., DEM) if needed and feasible.

Recent developments in ECoLaSS are based on both, optical and SAR data and led to improved/new status layers at $10 \mathrm{~m}$ and associated change layers at $10-20 \mathrm{~m}$. Thematic topics on the forest domain include improved status layers of Tree Cover Density (TCD) and Dominant Leaf Type (DLT) for the years 2017 and 2018 associated to the respective Tree Cover Masks (TCM), as well as an associated change 2017-2018 (Moser et al. 2018a). Regarding the grasslands, improved 2017 and 2018 status and change layers (Moser et al. 2018b), and a novel product on the use intensity (i.e., extensively/intensively management) based on the number of mowing events throughout the year are contained in the ECoLaSS portfolio. Linked to the new products developments in the agriculture domain, status layers 2017 and 2018 (i.e., crop mask) and crop types are generated, for which panEuropean representative categories are being defined and tested (Moser et al. 2018a, Moser et al. 2018b). To complement categorical maps (i.e., binary masks), and responding to the demands of generic and cross-cutting novel products allowing for multipurpose applications, biophysical variables and phenology indicators are applied in examples of crop status monitoring (e.g., intraseasonal start, peak and end of phenological activity derived from vegetation activity intermediate products). Illustrations here presented of higher-level continuous layers adding details into the land cover presence mask are the Tree Cover Density in forest, and the number of detected mowing events in grasslands.

This paper introduces the ECoLaSS project setup, embedding an overview of the collected user and stakeholder requirements, developed processing methodologies and the candidate prototypes for operational service implementation. The focus is laid on the established prototypes for potential next-generation HRL products on Forest, Grassland and Agriculture, based on dense Sentinel1 and Sentinel-2 time series analytics.

\section{MATERIALS AND METHODS}

The following selected innovative improved or novel products presented in this work are being developed, tested and prototypically demonstrated: Forest, Grassland and Agriculture layers. The area of study, data sources and methods are described in this section.

\subsection{Area of study}

Throughout Europe, representative demo sites of approx. $60,000-90,000 \mathrm{~km}^{2}$ size each were selected, covering boreal, Mediterranean, steppic, Atlantic, alpine and continental parts of 14 European countries, with good access to specific in-situ data, and representing the spread of biogeographic regions in Europe.
European sites cover parts of France, Belgium, Spain, Andorra, Sweden, Germany, Austria, Switzerland, Italy, Bulgaria, Serbia, Macedonia, Kosovo and Greece, as shown in Figure 1.

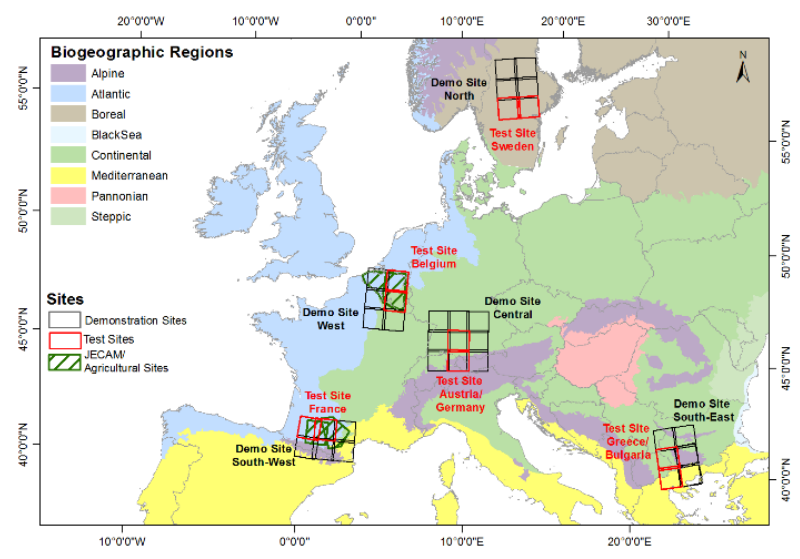

Figure 1. ECoLaSS Sites distribution in Europe

Forest layers are prototyped in the Central (Austria/Germany/Switzerland), North (Sweden) and South-East (Bulgaria, Serbia, Macedonia, Kosovo and Greece) demonstration sites and consist of a set of improved status layers for 2017/2018: Dominant Leaf Type and the continuous-scale Tree Cover Density product (Moser et al. 2018a). The basis for these layers is an improved tree cover detection, resulting in a binary Tree Cover Mask (TCM), which is used to generate an incremental update in form of the Tree Cover Change (TCC) layer 2017-2018. The grassland masks presented in this paper are produced in the Central demo site: status layers 2017 and 2018 (GRA) and grassland change layer 2017-2018 (Moser et al. 2018b), whereas the Grassland use intensity is tested in a smaller site within the Central demo site. For both forest and grassland, an additional change detection between the HLR2015 and the mask 2017 prototypes is assessed. Also under production, the grassland status and change prototypes are located in the South-East and West (Belgium, France) sites. As for agriculture, the crop mask 2017 and 2018 and the crop types 2017 and 2018 presented are also produced in the Central demo site (Probeck et al. 2019, Moser et al. 2018a,b). Two other prototypes will be produced in West (France/Belgium) sites in order to cover different biogeographical regions.

\subsection{Data sources}

Time series of optical (Sentinel-2) and SAR (Sentinel-1) satellite data of 2017 and 2018 are subject to pre-processing workflows to optimize geometric and radiometric consistency of the spectral bands, for which a digital elevation model (DEM) is necessary. Indices are derived from the resulting spectral bands. Several ancillary data sources are employed for the classification (e.g. LUCAS 2018, LPIS/IACS, ESA DWH VHR), be it for algorithm training or validation purposes.

\subsection{Methods}

From a methodological point of view, the temporal analysis techniques applied make use of a multitude of temporal 
feature descriptors (multiple temporal statistical metrics). Temporal features describe distinct spectral, temporal and phenological properties. By selecting suitable time windows, the mapping of phenological transition points and phases is possible by simultaneously mitigation of cloud cover issues within optical data.

\subsubsection{Pre-processing:}

In the case of optical data, atmospheric correction, topographic normalization and cloud and cloud shadow detection and masking are the key pre-processing steps. The ESA Sen2Cor package (Louis et al. 2016) and python scripts are used for this purpose. Cloud and cloud shadow masking embeds the generation of a binary mask, which is derived by a dedicated filtering and buffering process from the original Sen2Cor Scene Classification Layer (SCL). In the South-East demo site, the MACCS/MAJA cloud masking algorithm (Lounjou et al. 2016) is preferred in view of test performance outcomes. The topographic normalisation implemented in the Sen2Cor software uses a $90 \mathrm{~m}$ DEM and creates slope, aspect and terrain shadow products. The output bands are resampled to $10 \mathrm{~m}$ using the cubic convolution method whereas the SCL is resampled using nearest neighbourhood. The three bands with $60 \mathrm{~m}$ spatial resolution (Bands 1,9 and 10) are omitted in the Level-2A output. Topographic normalisation is performed to reduce illumination effects caused by certain topographic conditions and contributes to obtain a better consistency in the time series (e.g. sun angles and shadows dramatically vary throughout the seasons).

From the surface reflectance products, the following spectral indices are derived:

- $\quad$ NDVI (Normalized Difference Vegetation Index) NDWI (Normalized Difference Water Index)

BRIGHTNESS (derived through summation of the values of the bands Green, Red, NIR and SWIR1)

- $\quad$ IRECI (Inverted Red-Edge Chlorophyll Index)

Together with the spectral information, these vegetation indices are the input for the subsequent thematic processing.

In turn, SAR time series are based on Level-1 products in Interferometric Wide swath (IW) mode and Level-1 Ground Range Detected (GRD). The pre-processing steps is performed with the ESA SNAP tool: orbit update (includes automatic precise orbits download), thermal noise removal, radiometric calibration generating a beta band, terrain flattening to gamma naught based on SRTM 1sec HGT, terrain correction using the same DEM generating a $10 \mathrm{~m}$ resolution product and the export of the scene in DN units. After the orthorectification of the images, a multitemporal speckle filtering has been applied using a $5 \times 5$ Frost filter. Python scripts are used in indices derivation: VV (Gamma naught), VH (Gamma naught), Normalized Difference VV/VH, Ratio VV/VH.

\subsubsection{Temporal features computation:}

Temporal feature descriptors are able to depict and quantify a surface's status and its phenological behaviour over time as well as to capture the intensity and significance of change information and time series related statistical properties. Thus, they constitute powerful input features for various classification or analysis tasks. Not being directly related to image acquisition dates, neither customised scene selection efforts nor prior knowledge of change event dates is required. Hence, feature descriptors can be flexibly computed from reflectance or derived index data.

For grassland, the time windows testing included the spring period (01.03-31.05), late winter to spring (01.01-01.06) and a larger period (01.01-30.11). In the case of Forest, tests included several time windows (e.g., 15.03-15.06). The selected time windows for the TCM and DLT is related to the growing season (e.g., 15.03-15.09). In turn, the periods tested in Agriculture were selected to capture the crop season key moments (e.g., 15.03-15.10).

Seven temporal feature descriptors represent standard statistical temporal metrics of a time series: maximum, minimum, mean, median, standard deviation, covariance and and percentiles $(10,25,50,75,90$, difference 90-10 and difference 75-25).

In parallel to the new feature calculation and analysis, a feature selection method is applied. The K-Fold Cross Validation method is based on a stratified $\mathrm{k}$ fold sampling integrated in the machine learning package. This sampling method splits the training and test dataset into a number of $\mathrm{k}$-folds. It clones the classifier in every iteration and produces accuracy figures and a new training and test set. The algorithm finally yields a combination of the features with the highest accuracy. This subset of features is used for the classification process.

\subsubsection{Classification:}

Classification was applied using different combinations of sensor data and time periods to benchmark their respective feasibility, effort and accuracy: Sentinel-2, Sentinel-1, and the combination of Sentinel-1 and Sentinel-2 temporal features.

Two independent sample datasets were used for the classification and validation. In the case of forest and grasslands, samples were automatically extracted from a combined HRL 2015 sampling layer, consisting of the $20 \mathrm{~m}$ Layers Dominant Leaf Type, Imperviousness, Grassland and Water and Wetness. In addition, LUCAS 2018 points filtered by the observation type 1 attribute and visually inspected if required, were also selected. To reduce the number of possible outliers and errors in these samples, the following filtering steps were applied to the sampling layer: reduction of edge effects and mixed pixels through negative buffering $(20 \mathrm{~m})$ of the HRL product classes (e.g., coniferous forest, imperviousness, water) and removal of patches smaller than 1 ha. A stratified random point sampling has been performed within the remaining area. Subsequently, potential outliers have been removed by statistical analysis and visual checks, if required. For the agriculture crop mask and crop types, as no pre-existing HRL was available, LUCAS 2018 and LPIS data were respectively, employed for sampling purposes, following selection procedures as aforementioned. The sampling set is split into the set used for training purposes and the 
validation independent sample set, in a 50\%-50\% proportion.

Using a random forest-based classification approach on several hundred temporal feature descriptors, a feature selection is performed to identify the most meaningful input features for classification, ensuring a high resultant product quality as well as a cost and time efficient processing with high accuracy. A probability layer indicating the percentage of reliability constitutes a pixel-based quality indicator byproduct.

In the case of the intensity layer, NDVI time series are used to derive the number of mowing events, that are clipped to the grassland mask and reclassified by defining the extensive use category when less than or equal to 2 mowing events are detected during the year.

A final post-classification filtering was based on a five minimum pixel count of connected patches (e.g., GRA as well as non-GRA), and case-wise application of elevation thresholds (e.g., Agriculture > $1700 \mathrm{~m}$ threshold). All patches smaller than five pixels (i.e., minimum mapping unit 5 pixels) were removed to close holes in grassland patches and remove very small patches.

Consistent with the TCM \& DLT, a continuous-scale Tree Cover Density product with values ranging from $0-100 \%$ is produced at $10 \mathrm{~m}$ spatial resolution using band-specific temporal features from Sentinel-2 and a multiple linear regression estimator. The pixel-based TCD product provides information on the proportional crown coverage per pixel in percent, whereas tree cover density is defined as the „vertical projection of tree crowns to a horizontal earth's surface“. Two different approaches have been used to generate the status layer Tree Cover Density 2018 using a linear regression estimator: a mono-temporal classification using a "best-of" scene approach and a multitemporal classification using band-specific temporal features for defined time windows. The latter one shows very promising results and is recommended for an operational roll-out on European level.

\subsubsection{Change detection:}

In view of a potential future HRL Forest Incremental Update layer, the delineation of forest change/loss is based on the comparison of a pre- and post-change tree cover mask (map-to-map change approach with subsequent MMU filtering, followed by a NDVI plausibility analysis of detected changes. The Incremental Update layer resulting thereof, hereinafter explicitly named as Tree Cover Change (TCC), compares the pre- and post-change mask (TCM 2017 and TCM 2018) to detect areas of change with a minimum mapping unit of 1 ha. Due to the very short time interval of mostly $<1$ year between the two masks, this layer concentrates on negative changes (loss) only. The methodology incorporates both, Sentinel-1 and Sentinel-2 data and provides more flexibility in areas of frequent cloud cover. This combination of a map-to-map comparison and change indicators derived from Sentinel-2 time series (e.g., minimum NDVI differences) of both years provides a more robust change detection than a common map-to-map change approach. The approach has been also applied (slightly modified) to the grassland change.

\subsubsection{Validation and benchmarking:}

A statistical validation is carried out on the basis of a stratified systematic sampling approach with area-weighted accuracy calculation. Unequal sampling intensity resulting from the stratified systematic sampling approach is accounted for by applying a weighting factor to each sample unit based on the ratio between the number of samples and the size of the stratum considered. The weighing factor is inversely proportional to the inclusion probability (i.e., the probability that a pixel will be included in the sample) of samples from a given stratum. Within a geographic stratum, the inclusion probabilities of all sample units are equal. To combine sample data over several strata, a weighted estimator of the error matrix is required to account for the different inclusion probabilities among strata. The estimation weight is the inverse of each sample unit's inclusion probability, and the proportion of area for each cell of the error matrix is estimated. Else, true map accuracies might result over or under estimated. Overall accuracy and class specific accuracies (user and producer accuracy) are computed for all thematic classes from the weighted sampling probability-corrected confusion matrix $\mathrm{Ci}, \mathrm{j}$ (for points classified into class i and validated in class j), and $95 \%$ confidence intervals are calculated for each accuracy. From these, the F1 score statistic is computed per class for the status layers and change layers, as the harmonic mean between precision (i.e., User accuracy) and recall (i.e., producer accuracy), where an $\mathrm{F} 1$ score reaches its best value at 1 (perfect precision and recall) and worst at 0 . In case of the TCD, the regression goodness-of-fit statistics are computed (i,e, i,e, Mean Absolute Error MAE, Root Mean Square Error RMSE and the coefficient of dtermination $\mathrm{R}^{2}$ ). The layers are also assessed qualitatively by visual inspection using VHR data.

\section{RESULTS AND DISCUSSION}

The overall thematic accuracies for all Grassland, Forest, and Arable Land layer prototype implementations produced so far are promising, ranging from $77-98 \%$. The lessonslearned from the first temporal feature descriptors extraction and selection tests, together with the added value of the combined use of both optical and radar data, lead now to refined workflows and results.

\subsection{Forest products}

To derive the 2018 TCM and DLT, in total, 234 features were available to feed the machine learning algorithm: 182 features for the Sentinel-2 indices and bands, and 52 features from the Sentinel-1 single bands and indices. SAR temporal features derived from the $\mathrm{VH}$ polarisation (e.g. VH_p025, VH_p010) turned out to have a high importance in the tree cover detection followed by features derived from the Sentinel-2 bands B02 and B03 as well as NDVI and NDWI features. With respect to the DLT classification, SAR features show no benefit for the leaf type discrimination. Here, band-specific features from Sentinel2 dominate clearly over all other derived features. Worth mentioning is the dominance of features derived from the SWIR bands (B11, B12). A significant influence of the feature selection method on the overall accuracy figures (retrieved by LUCAS 2018 points) could not be observed. 
Increasing the spring time window (15.03-15.06) to the extent of the whole growing season period (15.03-15.09) is drastically increasing the data volume and processing time (and logically also the production costs) but has a positive effect on the achieved overall accuracy of the TCM, which is in the magnitude of 2 to 4 percentage points. This is mainly due to the increased data availability and a potentially higher rate of data acquisitions without or with less cloud cover. However, the strongest influence on classification accuracies can be observed by the quality of the samples. A subsequently performed validation with 800 LUCAS 2018 points confirmed very high overall accuracies for both, the TCM as well as the DLT classification (98\% OA and 93\% OA respectively in the Central demo site).

In order to generate a comparable and future-oriented data basis for the incremental update, the same feature and sample sets have been used to derive improved TCMs and DLT layer for the reference year 2017. Then, the incremental update layer TCC 2017-2018 has been produced following a map-to-map change approach, leading to an improved geometric and thematic accuracy of the derived changes (forest loss).

The continuous Tree Cover Density product 2018 (Figure 2) adds unprecedented detail to the binary Tree Cover Mask. For the TCD, the mean and median features from the spectral Sentinel-2 bands provide promising results. Both classification approaches tested in the forest status layers rendered similar results (best-of-scene and mono-temporal), although in the end the multi-temporal classification using band-specific temporal features for the defined time windows was selected. The goodness-of-fit statistics for the linear regression model of the TCD 2018 obtained are: 7.65 MAE, 8.14 RMSE, and $0.93 \mathrm{R}^{2}$.

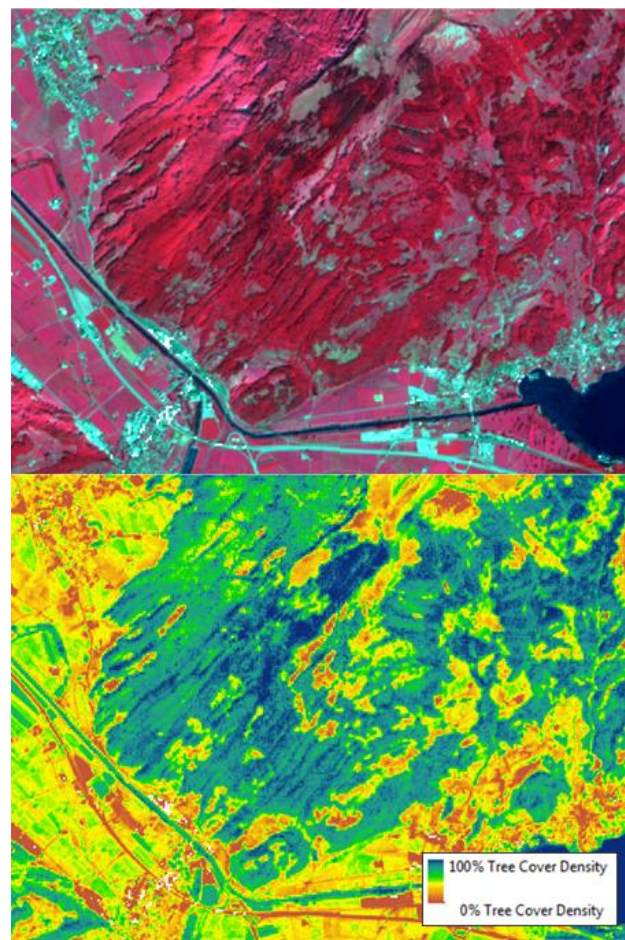

Figure 2. Multi-temporal S-2 feature stack (upper figure) and the derived TCD 2018

\subsection{Grassland products}

With the availability of dense optical and SAR time series from Sentinel-1 and Sentinel-2, grassland mapping can profit from the increased information content provided by temporal measurements of the reflectance of grassland areas over the year. Based on reinterpreted LUCAS samples a supervised classification approach using the Random Forest classifier has been successfully applied.

The feature selection tests automatically executed by the Random Classifier have shown that, in the Central demo site, best performing input indices are NDVI, NDWI, IRECI and Brightness. Regarding SAR data generally the following annual coefficients have shown the best performance: $\mathrm{VH}$ polarisation, the $\mathrm{VV}$ polarisation and the NDVVVH Ratio.

The later winter to spring (January to June) time window in the end did not bring an added value for classification. In different biogeographical regions the short time window can differ (which is also the case in the forest and agriculture products). To better match the local conditions, it is important to define a time window where grassland and cropland (class generating most misclassifications) are best separable (e.g., define the period when grassland is already greening whilst cropland is not). In Mediterranean regions, for instance, this window may be shifted more towards winter (e.g. Dec-Mar).

After the visual interpretation of all classifications, it can be observed that using optical data only more confusion between grasslands and cropland are present, whereas using SAR data only more misclassifications between grassland and roads are present. The combined approach shows more homogenous patches than using SAR data only, diminishing confusion with other classes suchlike tree cover and plantations, that otherwise cannot be excluded when using only S-2. This is relevant as in fact the overall accuracy values obtained are misleading while the visual inspection demonstrates the combined approach performs best. A main requirement however is the precise preprocessing of the dense time series including a topographic normalisation for hilly to mountainous terrain. For SAR time-series the application of multi-temporal filtering on gamma naught corrected imagery is recommended

For the Central site Grassland mask 2018, the overall accuracy is $96.6 \%$ ( $0.93 \%$ Confidence Interval), producer accuracy is $91.0 \%$ and user accuracy $90.5 \%$.

Regarding the change layers, as only the geometrical change areas are shown and no further exclusion of nonchange by the usage of e.g. NDVI minimum or other change indicators is applied to the 2017-2015 change layer, there are many more changes visible compared to the 2017/18 change layer. Most of them in truth are no real change, instead resulting from weaknesses in the 2015 layer.

In areas of higher elevation and where snow cover is found for long periods of the year in the South of the demo site, the classification is not that accurate for both years $(2015 \&$ 2018) which in turn leads to greater differences and 
therefore change detection which is basically no change. An elevation threshold was applied to reduce such errors, which significantly improved the respective grassland masks in the most problematic regions. Filtering significantly contributes to keep meaningful changes while removing small areas, likely classification errors. All areas below 1 ha (25 pixels) were filtered.

The figure below shows a detail of the grassland intensity 2018 layer, consistent as derived from the areas defined as grassland within the grassland status layer, and based on a simple although effective NDVI time series analysis, with a good trade-off between computer resources and preprocessing timing (i.e., impact in updates production timeliness) and achieved thematic accuracy. The datasets used for validation for the use intensity grassland product are the IACS/LPIS, generating an overall accuracy of $81.5 \%$.

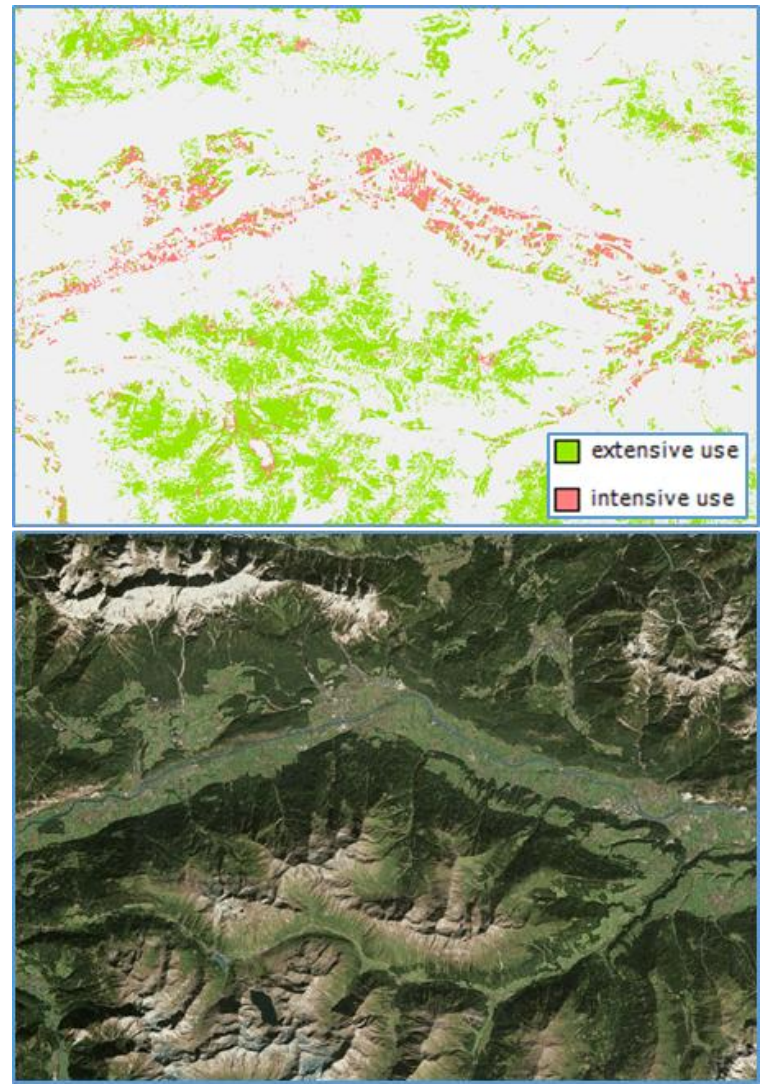

Figure 3. Grassland intensity layer 2018 (upper figure) compared to Bing Maps Aerial of the same region

The current temporal density of optical time-series from Sentinel-2 for the years 2017 and 2016 restricted the applicability of time series methods, as reflectance trajectories depend on the grassland dynamics over the vegetation period such as e.g. mowing events. The high cloud coverage in 2018 in central Europe limits the method of comparing NDVIs of consecutive acquisition dates: mowing events may not be detected in areas covered by clouds for long periods. Filtering improved look and feel by reducing noise. For the use intensity layer, a filter of 4 pixels in size was applied. All areas within the grassland mask where filtered, so that there are no intensity classes smaller than 5 pixels in the end. Within small grassland patches, it might happen that e.g. three pixels are categorized as extensive and two are intensive. In such cases, the filter would cause the class values to jump between classes with each filter iteration without getting a patch of five unique values. If so, the patch was filtered in favour of intensive use because most of the areas are used intensively in the demo site.

\subsection{Agriculture Products}

The time interval algorithms are strongly affected by undetected clouds/cloud shadows as well as confusion with bright surfaces in the cloud mask. These algorithms present many artefacts and data gaps due to the short compositing period and the interval between images available in the time series. To avoid potential artefacts derived from the presence of unmasked cloud cover pixels, time periods were selected to guarantee a sufficient number of imagery to minimize the distortion that extreme values would pose to the statistics.

Concerning Agriculture classification an initial set of criteria to evaluate the best compositing method for crop recognition (cropland-CL, crop type-CT) and crop growth monitoring (CG) have been selected. The benchmark is performed on Central (Germany) and Belgium site and show promising accuracies and high potential of time series and derived temporal features for crop mask extraction and crop type monitoring. As is not uncommon in agriculture products, the local conditions (e.g., climate, soil, altitude, topography, crop phenology) influencing this specific land cover makes it a challenge to target a HRL at a continental scale. In our case, stratification was applied for a better adaptation to the particularities of the different subregions within the Central demo site.

In Central tests, LUCAS data from 2018 constitute the main part of the sample base and additional samples for forest, grassland, water and urban areas have been taken from HRL2015. The availability and representation of crop and specifically crop types samples is essential for the model training and significantly impacts the performance and quality. Results point out to the fact that the added value of using S-1 data is dependent on density of optical time series: e.g., due to the positive data situation in 2018 - high number of optical and cloud free imagery - the additional benefit of using S1 is low. This fact is also represented in the accuracy figures for the experiments with S-1 and S-2 only. The combined S-1 and S-2 data crop mask 2018 stratified classification (i.e., accuracy figures computed separately for each stratum) provides $\mathrm{OA}$ in the range of 98-99\% and F1Score ranges between $98-99 \%$ for non cropland and $89-91 \%$ for cropland (user and producer accuracies respectively $97-98 \%$ and $98-99 \%$ for noncropland and $90-94 \%$ and $88-90 \%$ for cropland). 


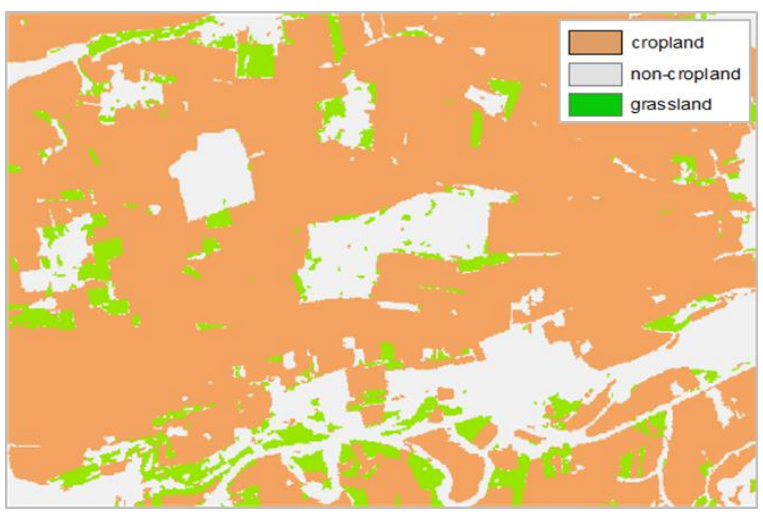

Figure 4. Crop mask 2018 (detail) together with the Grassland mask 2018

Different methods are applied for crop type classification where S-2 data sets and integration of S-1 and S-2 data are used for calculating input features. Unlike the other land covers addressed, in the agriculture domain, the temporal features contribution differs between the crop mask, crop types map and for each, within the strata. In the Central demo site, it could be stated that in the crop mask around $2 / 3$ selected features come from S-2 (from B8 and B12, also minimum, mean, maximum, standard deviation, and percentiles, NDVI, Brightness, NDWI), and 1/3 from S-1 (minimum, mean, maximum, standard deviation). Analogously, in the crop mask the same features derived from $\mathrm{S} 2$ are selected and in this case the $\mathrm{S} 1$ contribution is of significantly minor relevance. It is worth mentioning that the used data are subjected to pre and post processing steps. The LPIS datasets, are used as reference data. The Random Forest (RF) classifier is selected due to the efficiency on large data bases, the ability of using thousands of input variables without deletion, estimation of the relative importance of the variables and the relative robustness to the outliers. Training and validation samples are derived from LPIS datasets. Specifically, the Crop Type map was calculated basing on LPIS data from Baden Württemberg and Austria. The majority filter is applied for harmonization of the results.

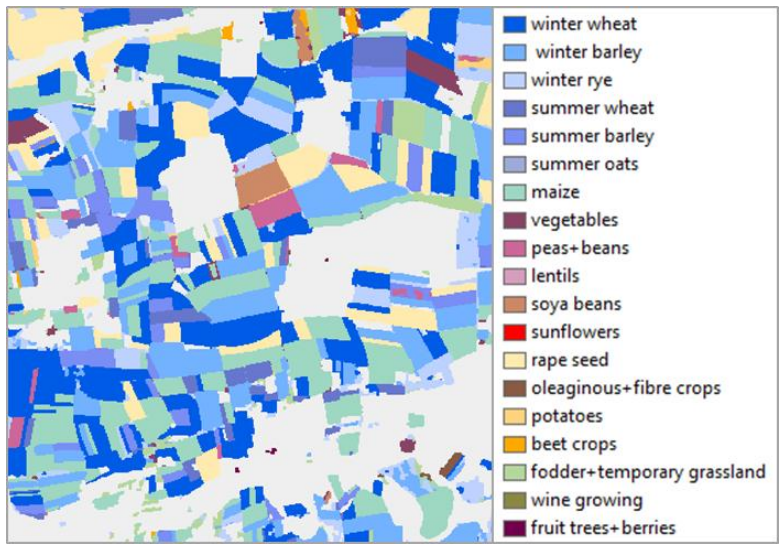

Figure 5. Crop types map 2018 (detail)

A newly arranged Crop Type class aggregation which is suitable to be used in a Pan-European context and at the same time adaptable to regional conditions to a certain extent has been used for the classification approach. The
Crop Type Legend is oriented towards the LUCAS class structure (aiming at the potential of LUCAS data being a source of information available in most EEA countries). It comprises the most common crop types for the crop groups of winter and summer cereals, vegetables, dry pulses and legumes, industrial crops, root/tuber crops, fodder crops and permanent crops. The legend comprised initially nineteen classes although in the end class aggregation was applied due to misrepresentation of minority classes in the samples and its effects on accuracy. The results strongly suggest that a sufficient number of high quality samples has a strong impact on the accuracy of the classification, both for the crop mask as well as for the crop type map (in the latter, only LPIS/IACS provide suitable attributes). Crop types which are not well represented in the sample base will lead to lower accuracies.

The combined S-1 and S-2 data classification (using the same technical workflow as for the Crop Mask) provides an OA of $86 \%$ and F1Score ranging from $69 \%$ for lentils to $98 \%$ for rape seed (differing for each of the crop types). All in all, five classes present a F1Score > 90\% and eight present a F1Score $>80 \%$.

In case of the prototype for the Central demonstration site further research is needed to optimize the validation methods both for the crop mask and the crop types, due to inconsistencies between the different ancillary data such as LPIS data of Baden Württemberg and those of Austria, in terms of naming and general class structure. Furthermore, improvements towards an enhanced separability of the crop types are being addressed. The provision of the LPIS data of Bavaria might support this in the Central demo site. Besides actions related to extending the sample base, one promising approach that has outperformed the look and feel of the crop mask is the stratification basing on biogeographic criteria. Still, the differentiation between the various grassland types, between managed grassland and agrarian grass types remains an issue and should be more deeply explored. More than with any other LC, the quality of agricultural products highly depends on additional information sources to define the suitable workflow.

Notwithstanding, the consistency between the HRL prototypes for Grassland, Crop Mask and Tree Cover Mask is satisfactory.

Besides quantitative statistical accuracy assessments and other quality metrics, a rigorous benchmarking procedure is applied to assess the prototypes' overall operational readiness and technical maturity for integration into the CLMS overall setup and architecture. This comprises assessing, amongst others, automation levels, portfolio complementarity, or the state of the art/innovation degree. Moreover, an integration plan for the most suitable prototypes will be set up.

\section{CONCLUSIONS}

Innovative methods for automated high volume data processing of Sentinel (optical and SAR) time series are being developed in the fields of Sentinel-1/-2 time series integration, time series pre-processing methods, thematic classification from time series analysis, change detection 
from time series analysis and incremental update methodologies for the Copernicus Land High Resolution Layers (HRLs).

With regards to accuracy, in addition to the time windows selection for time features computation, sampling is key. In ECoLaSS, a multi-stage sampling approach has been applied: automatic reference sampling based on a sampling layer generated from the HRLs 2015, outlier detection with visual/statistical validation of the samples and split of the sample dataset into training and validation dataset, initial classification and re-sampling based on an analysis of omission and commission errors with subsequent iteration loops.

Iterative calculation over a time stack allows for implementing suitable dynamic change detection systems, which require to be frequently updated, such as it would be the case for the existing HRL forest and grassland services as well as for a potential future arable land service. The change layer products quality is totally relying on the quality of the respective status layers. The probability layers for each of the status products from which the change is assessed by indicating the pixel level accuracy confidence and the expert knowledge are relevant in a suitable production workflow for a comprehensive change detection.

The improvement endeavours of existing products comprise assessing prototypes of enhanced tree cover, leaf types, tree cover density within, and tree cover change layers as yearly updates, as well as better-quality permanent grassland classification and use categorisation together with a yearly change identification approach. The production of an Incremental Update Layer for the Forest HRL and its rollout on European level has been rated as feasible.

Entirely novel products are targeted, such as a possible future HRL on arable land, for which a prototype is presented, targeting a pan-European crop mask of high precision and a representative, robust classification of crop types.

The prototype of the new agriculture service shows that the definition of a pan-European crop-types legend is not straightforward, as crop phenology follows rather regional and local scale patterns and not all classes are present at the continental scale. In addition, the availability of a sufficient number of high quality samples is essential to obtain reliable crop masks and crop types products within. The demo site in Central Europe proves that a locally-adapted stratification accounting for biogeographic heterogeneity (i.e., climate, soil, altitude) highly reduced misclassifications for both agricultural products.

In ECoLaSS, these and all other candidate prototypes are being produced with a view to a potential pan-European for service roll-out. For the most promising prototypes a detailed integration plan into the Copernicus service architecture is being developed.

\section{ACKNOWLEDGEMENTS}

The research leading to these results has received funding from the European Union's Horizon 2020 Research and Innovation Programme, under Grant Agreement no 73000. The content of this paper does not reflect the official opinion of the European Union. Responsibility for the information and views expressed therein lies entirely with the author(s). The study used Copernicus Land Monitoring Service products (C) European Union, CLMS 2018, European Environment Agency (EEA). We acknowledge the use of LPIS data provided by the agricultural ministry of Baden-Württemberg and LUCAS 2018 provided by the European Commission/EUROSTAT.

\section{REFERENCES}

Lonjou, V., Desjardins, C., Hagolle, O., Petrucci, B., Tremas, T., Dejus, M., Makarau, A., Auer, S., 2016. MACCS-ATCOR joint algorithm (MAJA). Presented at the Remote Sensing of Clouds and the Atmosphere XXI, International Society for Optics and Photonics, 1000107.

Louis, J., Debaecker, V., Pflug, B., Main-Knorn, M., Bieniarz, J., Mueller-Wilm, U., Cadau, E., Gascon, F., 2016. Sentinel-2 Level-2 Processor Sen2Cor

Moser, L., Probeck, M., Ramminger, G., Rieke, C., Mack, B., Ickerott, M., Storch, C., Sommer, C., Richter, R., Herrmann, D., Ruiz, I., Kovatsch, M., and Schwab K. (2018a): Evolution of Next-generation Copernicus High Resolution Layers on Forest and Agriculture: The ECoLaSS Project. $3^{\text {rd }}$ joint EARSeL LCLU \& NASA LCLUC Workshop (11-12 July 2018 in Chania, Greece)

Moser, L., Probeck, M., Ramminger, G., Richter, R., Herrmann, D. (2018b): Sentinel Time Series for Nextgeneration Copernicus High Resolution Layers on Agriculture and Grassland. INSPIRE Conference (18-21 September 2018 in Antwerp, Belgium)

Probeck, M., Schwab, K., Sevillano Marco, E., Herrmann, D., Sandow, C., Richter, R., Moser, L.: Prototypes of Future Copernicus Land Monitoring Products: The ECoLaSS Project. ESA Living Planet Symposium (13.-17. May 2019, Milano, Italy) 